\title{
The current advances of CRISPR/Cas-based systems for the detection of COVID-19
}

\author{
Sasan Ghaffari1,2,3, Hanif Kazerooni ${ }^{1, *}$, Amir Salehi-Najafabadi3,4,* \\ ${ }^{1}$ Department of Science and Technology, Supreme National Defense University, Tehran, Iran \\ 2Department of Hematology, School of Allied Medical Sciences, Tehran University of Medical Sciences, Tehran, Iran \\ 3 Research Center for New Technologies in Life Science Engineering, University of Tehran, Tehran, Iran \\ 4 Department of Microbiology, School of Biology, College of Science, University of Tehran, Tehran, Iran
}

\begin{abstract}
The novel coronavirus disease 2019 (COVID-19) still poses a serious threat to every human on the planet nearly 1.5 years after its beginning. Unfortunately, the current diagnostic methods, although highly sensitive and specific, still suffer from many shortcomings. Faster and easy-to-operate diagnostic systems are undoubtedly sorely needed. The CRISPR/Cas platform has gained much attention in recent years in a wide range of biomedical sciences. Besides its treatment potential and drug and vaccine development, it can be used for disease diagnosis. Thus, the CRISPR/Cas-based system holds great promise for providing a rapid and easily deployable COVID-19 detection and is on par with the conventional diagnostic approaches in terms of sensitivity and specificity. In this review article, we discuss the latest advances of CRISPR/Cas technology as a fast and reliable severe acute respiratory syndrome coronavirus 2 (SARS-CoV-2) detection method.
\end{abstract}

Keywords: COVID-19, SARS-CoV-2, Diagnosis, CRISPR

\section{Introduction}

The emergence of the new severe acute respiratory syndrome coronavirus-2 (SARS-CoV-2) in December 2019 in Wuhan, China marks a major turning point for mankind. The health-related and economic issues associated with the devastating coronavirus disease 2019 (COVID-19) quickly swept across the globe while threatening the lives of billions with a global pandemic. The disease has taken the lives of millions and shows no signs of slowing down even with the advent of vaccines. The alpha (229E and NL63) and beta (OC43 and $\mathrm{HKU} 1$ ) groups of the coronavirinae subfamily can infect humans; two other beta group viruses, Middle East respiratory syndrome (MERS$\mathrm{CoV}$ ) and severe acute respiratory syndrome (SARS$\mathrm{CoV}$ ), were previously endemic to humans, none of which have caused such destructive effects $[1,2]$. These viruses primarily infect the respiratory tract cells that carry angiotensin-converting enzyme II (ACE2) and subsequently other cells that express ACE2.

\footnotetext{
"Corresponding author:

Hanif Kazerooni, Ph.D

Department of Science and Technology,

Supreme National Defense University, Tehran, Iran

Tel/Fax: +98910 2118860

Email: kazeroonihanif1970@gmail.com

https://orcid.org/oooo-0002-7159-1237
}

Amir Salehi-Najafabadi, Ph.D

Department of Microbiology, University of Tehran,

P.O. Box 11155-4563, Tehran, Iran

Tel/Fax: +98912 2984877

Email: amirslehi@ut.ac.ir

https://orcid.org/oooo-0oo2-3661-4915

Received: August, 08, 2021

Accepted: September, 20, 2021
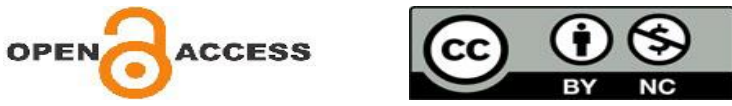
Ultimately, the unhinged virus replication and the uncontrolled inflammatory response of the immune system result in acute respiratory distress syndrome (ARDS), multiple organ failure, and death [2].

Besides finding a potential treatment for COVID19 , early and specific detection of the virus are crucial in breaking the transmission cycle and stopping the disease from spreading. The positive-sense RNA of SARS-CoV-2 is quite similar to that of SARS-CoV and is comprised of up to 14 open-reading frames (ORFs). ORF1a and ORF1b encode 16 non-structural proteins and constitute the vast majority of the genome. At the 3 ' end of the genome, the structural proteins include spike (S), membrane (M), envelope (E), and nucleocapsid (N) [3]. Quantitative real-time reverse transcriptase-polymerase chain reaction (qRT-PCR) is currently the standard method recommended by the world health organization (WHO) for specific detection of the mentioned SARS-CoV-2 genome sequences. However, due to various reasons such as being time-consuming, requiring rigid sample preparations and trained operators, and having stationary equipment, wide-range use of RT-PCR is somewhat difficult. Also, in some cases, the sensitivity of the method is reported to be as low as $42.1 \%[4,5]$, which calls for the introduction of more sensitive and versatile detection systems. Clustered regularly interspaced short palindromic repeats (CRISPR) and its associated protein (Cas) have been the focal point of advanced gene-editing since this platform is much more flexible and precise than its predecessors. Considering the emergency of COVID-19, scientists have focused on repurposing CRISPR/Cas system toward a diagnostic and even therapeutic approach [6]. In this article, we review the current SARS-CoV-2 detection methods and introduce various CRISPR/Cas-based approaches to virus detection.

\section{SARS-CoV-2 routine detection methods}

Currently, the most widely used method of detecting SARS-CoV-2 involves nucleic acid (NA) amplification and subsequent detection. NA detection methods have proven successful in the past with MERS and SARS-CoV outbreaks [7], and constitute the current standard SARS-CoV-2 detection method as RT-PCR, a variant of the PCR method. In RT-PCR, the sample is collected, lysed, and its RNA extracted. The purified RNA is reversely transcribed to complementary DNA (cDNA) and amplified. The detection of certain regions of the $\mathrm{cDNA}$, such as $\mathrm{N}, \mathrm{S}$, and Orfi genes, equals virus detection and SARS-CoV2 infection. The sensitivity of RT-PCR greatly depends on the chosen primer/probes [8]. Using unspecific primer/probe sets results in no detection of virus in low load (cycle threshold $>30$ ), or unspecific detection of other coronaviruses (hCoV-OC43, hCoV-229E, and MERS-CoV) due to cross-reactivity [9]. Unfortunately, the RT-PCR method is dependent on expensive instruments and trained laboratory scientists and is a lengthy process that is in contrast to create rapid, highly sensitive, and point-of-care (POC) diagnostic systems.

Over the years, other prominent PCR-based methods have emerged as alternatives to RT-PCR. Digital PCR (dPCR) and reverse-transcription loopmediated isothermal amplification (RT-LAMP) are to name a few. In dPCR, the sample is separated into discrete reactions and each is carried out separately. dPCR does not rely on a standard curve. It can detect much less viral load and it is less susceptible to amplification inhibitors [10]. LAMP is a step forward toward more rapid and POC testing which is crucial in the current pandemic. The method amplifies NA using 6 specific primers and a DNA polymerase enzyme with chain displacement activity. Such an activity circumvents the need for DNA denaturation via heat. Thus, the reaction may be carried out at one constant temperature (isothermal) making the process simpler and the device less intricate. If the starting NA is RNA, reverse transcriptase is added to create cDNA (RTLAMP) (Table 1) [11]. Reverse transcription recombinase polymerase amplification (RT-RPA) is another isothermal amplification method that works on the same basis as RT-LAMP and eliminates the need for thermal cycling machines.

All in all, accurate diagnosis of COVID-19 in suspected individuals is imperative to hinder the spread of the virus. The sensitivity and specificity of the current diagnostic methods are crucial in drawing an accurate comparison between each platform. The specificity and sensitivity of NA amplification methods depend on various conditions. While targeting the Orfi, E, and RdRP genes produce accurate results, $\mathrm{N}$ gene detection decreases sensitivity [12]. The contamination of the specimen has shown a decrease in the sensitivity of RT-PCR [13]. A study on 194 samples compared dPCR and RT-PCR and reported higher overall specificity and sensitivity of dPCR 
Table 1. The most widely used SARS-CoV-2 diagnostic tools

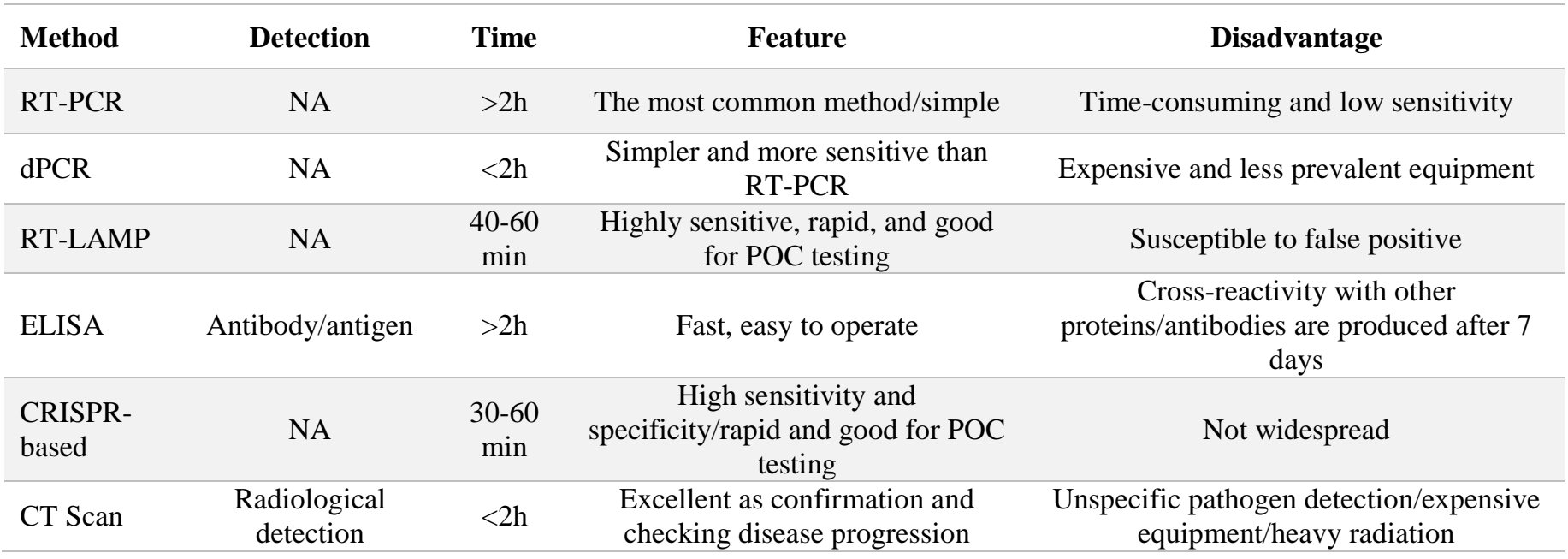

NA: nucleic acid; CT; chest tomography; ELISA: enzyme-linked immunosorbent assay

compared to RT-PCR [14]. These results were further confirmed by another study on 77 clinical samples, especially on low viral loads [15]. RT-LAMP provides the same level of specificity and sensitivity as RT-PCR and also has the added benefit of not requiring a thermal cycler. Serological methods, such as enzymelinked immunosorbent assay (ELISA) have the advantage of being rapid and high throughput; however, they cannot be designated as a gold-standard method since antibody response and its clinical significance in COVID-19 is not yet fully understood [16]. They are not as sensitive as nucleic acid-based techniques. In CRISPR/Cas platforms, the specificity is guaranteed by Cas enzyme activation after CRISPR RNA binds to the target sequence. Signal emission after the cleavage of the labels, on the other hand, ensures sensitivity. In a study by Hou et al. on 52 samples, the CRISPR platform showed 100\% specificity and sensitivity compared to the sensitivity (90.4\%) and specificity (89.2\%) of RT-PCR [17]. Depending on the detected gene, the CRISPR method had the same or higher sensitivity and specificity compared to RT-PCR in another study [18]. Considering its numerous advantages discussed below, CRISPR could potentially revolutionize the way we fight invading pathogens, such as SARS-CoV-2.

\section{A primer on CRISPR/Cas}

In response to the disastrous outcome of the COVID-19 pandemic, it is crucial to push forward the detection capabilities of laboratories worldwide. Early detection of the virus is crucial since it allows for rapid clinical intervention and stops the unsuspecting infected carrier from spreading the virus. CRISPR was first discovered in the 1980 s as a part of the immune system of prokaryotes against the invasion of viruses and plasmids [19]. The system essentially works on the RNA-guided catalytic activity of Cas proteins. Apart from CRISPR/Cas sensational reception in the field of gene editing, it has gained much attention as an NA detection platform due to its efficacy and simplicity. The two major constituents of CRISPR/Cas are a single guide RNA (gRNA) that recognizes the target sequence and a Cas endonuclease that proceeds to cleave the target sequence. The gRNA itself is made up of trans-activating RNA (tracrRNA) and CRISPR preRNA (crRNA) which recognizes a small 3-8 bp DNA sequence called proto space adjacent motif (PAM). CRISPR/Cas essentially works like molecular scissors and based on the Cas protein, it is divided into two classes and each of them consists of three types. Class 1 is type I, III, and IV, while class 2 includes type II, V, and VI [20]. Class 2 CRISPRs are widely used in preclinical and clinical studies. CRISPR/Cas9 of class 2, type II is used to cleave double-stranded DNA (dsDNA) to delete or insert certain genes in somatic cells (Table 2). This method has shown great promise in clinical trials of $\mathrm{T}$ cell therapy for cancer treatment [21]. Other types of class 2 CRISPR systems are also demonstrating the potential for genetic manipulation 
and diagnosis of infectious diseases that will be discussed later.

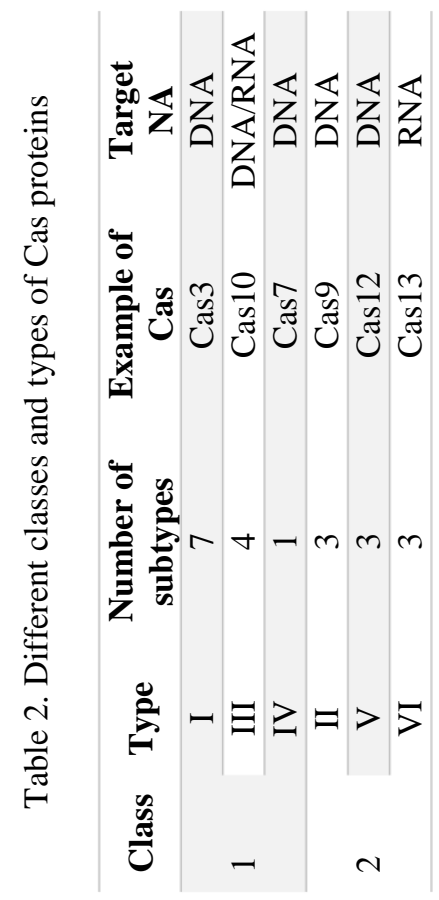

\section{CRISPR-based detection methods}

RT-PCR is currently the most prevalent mode of virus detection, including SARS-CoV-2. Still, other methods of virus detection are being explored because of some challenges associated with RT-PCR. Difficulties of costs, availability, time, the need for trained laboratory scientists, and false negative with low viral load or emerging SARS-CoV-2 variants are some instances of RT-PCR shortcomings [22]. Regardless of its recent introduction as a gene-editing platform, the novel CRISPR/Cas system is capable of detecting virus-containing samples. The platform has been used for the detection of viruses, such as the dengue virus (DENV) and Zika virus (ZIKV). CRISPR platform's potentials in virology were first introduced in 2018 by Myhrvold et al. reporting the capacity of the platform to detect 1 copy of the virus in samples of ZIKV and DENV [23]. This prompted the scientific community to manipulate different aspects of the platform to develop approaches for virus detection and eradication. Various CRISPR-based diagnostic (CRISPR-Dx) systems can be used as a stand-alone platform or combined with other established methods to provide a rapid and precise SARS-CoV-2 detection system.

\section{CRISPR/Cas12-based SARS-CoV-2 detection}

A novel technique called SARS-CoV-2 DNA endonuclease targeted CRISPR trans reporter (DETECTR), and it was recently reported to detect SARS-CoV-2 RNA in samples as low as 10 virus copies/ $\mu \mathrm{l}$ in less than 40 minutes. The technique utilizes CRISPR/Cas12a in tandem with RT-LAMP [24]. The RT-LAMP performs the reverse transcription of the virus RNA into cDNA. Then, the crRNA of CRISPR which is designated to recognize certain virus sequences binds to target DNA allowing for Casi2a to cleave the single-stranded target DNA (ssDNA). Cas12a subsequently cleaves the reporter molecule, and the resultant fluorescent signal is captured via lateral flow strips to indicate the presence of the virus (Figure 1). Ding et al. reported the development of the all-in-one dual CRISPR/Cas12a (AIOD-CRISPR) system for rapid, ultrasensitive, visualized detection of SARS-CoV-2 and HIV viruses [25]. AIOD-CRISPR uses two crRNAs for a more specific DNA recognition, and all the required components for NA amplification are mixed into a single reaction. Based on the color change in the reaction mix, the results can be interpreted with the naked eye. CRISPR/Cas12a-based assay with a naked eye readout (CRISPR/Cas12a-NER) is another similar model of rapid SARS-CoV-2 detection. The system can detect 10 copies $/ \mu$ in a sample under 40 minutes and uses crRNA as a recognition tool and ssDNA as a reporter. In the presence of the virus, Cas12a also cleaves the reporter molecule which emits a green fluorescent color in $458 \mathrm{~nm}$ and it is conceivable by the naked eye [26].

Further increases in the sensitivity of CRISPR/Cas12 systems, Huang and colleagues developed the CRISPR-fluorescence detection system (CRISPR-FDS) method that detects viral genome of two copies in a sample in under 50 minutes after the amplicons are produced using RT-PCR or RPA. Although CRISPR-FDS is unable to quantitatively determine virus titers, the system is much more sensitive than the conventional RT-PCR [27]. By coupling RT-LAMP with CRISPR/Cas12, another team created iSCAN (in vitro Specific CRISPR-based Assay for Nucleic acids detection) to detect SARS$\mathrm{CoV}-2$ infection in 60 minutes. The system is aimed at low-resource areas and it has claimed that the reagents can be produced locally [28]. Cas12b-mediated DNA 


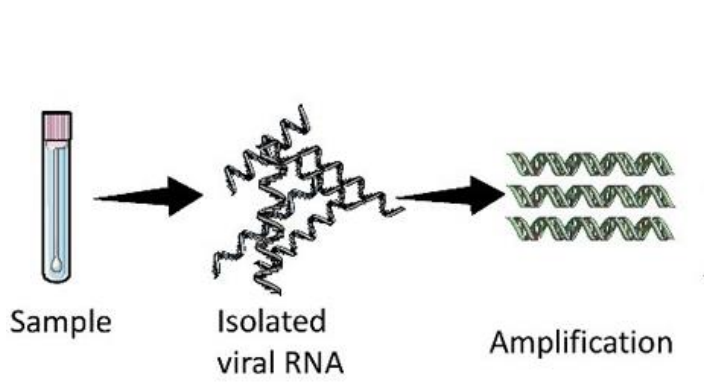

viral RNA
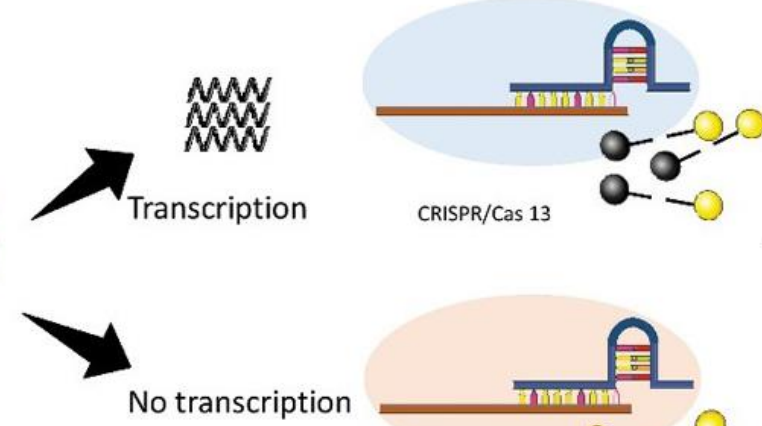
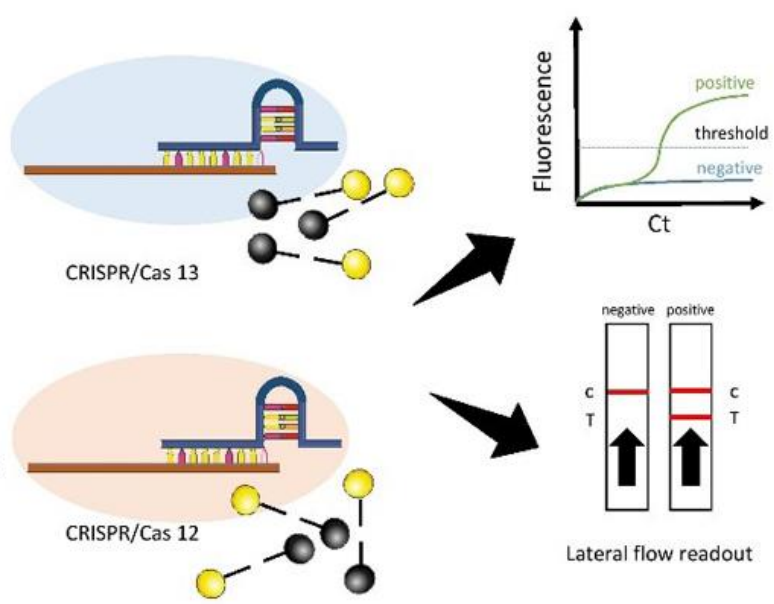

Lateral flow readout

Figure 1. A schematic of the protocols for SARS-CoV-2 detection using CRISPR/Cas. The viral RNA is first extracted, purified, and amplified into dsDNA by RT-RPA or RT-LAMP. For SHERLOCK, the DNA is first transcribed to ssRNA followed by recognition of CRISPR/Cas13a and cleavage of reporters. In the case of DETECTR, the amplified DNA is recognized by CRISPR/Cas1, and the cleavage of reporters produces signals. The emitted signals are picked up and visualized either by lateral flow readouts or fluorescence devices (Figure illustrated with the help of https://smart.servier.com/).

detection (CDetection) uses Cas12b for DNA cleavage and it is reported to be more sensitive than DETECTR (Cas12a) and SHERLOCK (Cas13) [29]. Other CRISPR systems of this category include One Hour Low-cost Multipurpose highly Efficient System (HOLMES), CRISPR-assisted detection (CASdetec), various nucleotide guard (VaNGuard), (STOPCovid), isotachophoresis CRISPR (ITP-CRISPR) [30].

One important aspect of any diagnostic method is its ability to be translated into clinical settings. DTECTR system, for instance, has shown such potential by being a convenient, rapid, and sensitive alternative to the traditional RT-PCR. A study on 378 COVID-19 patients compared the sensitivity of RTPCR and DTECTR and found both to be equally sensitive which is a positive point for DTECTR [31]. Another important aspect of the CRISPR-based method illustrated in this study is the capacity of the system to detect multiple SARS-CoV-2 genes, such as $\mathrm{N}$ or Orfi. Hence, if one gene is mutated and undetectable, the presence of the other gene will correctly diagnose the sample as positive. To further decrease the test time (to $30 \mathrm{~min}$ ) and increase the automation, microfluidic systems are being developed to automatically extract the RNA of SARS-CoV-2 samples, and control and accelerate the process using electric field gradient [32].

\begin{abstract}
6. CRISPR/Cas13-based SARS-CoV-2 detection

SCRISPR/Cas13 like CRISPR/Cas12 systems depends on collateral cleavage, meaning that after the protein binds to its designated NA sequence, it starts cleaving its surrounding single-stranded NA indiscriminately. Thus, the probes which consist of quencher and fluorophore are cut, and a fluorescence signal is emitted because of the distance between them. Specific high-sensitivity enzymatic reporter unlocking (SHERLOCK) is an ultrasensitive system of detecting SARS-CoV-2 that similarly to DETECTR depends on the RT-RPA for reverse transcription and amplification of viral RNA into dsDNA. Then, after T7 transcription into single-stranded RNA (ssRNA), CRISPR/Cas13a enzymology is responsible for the cleavage of the RNA. To minimize the need for extraction equipment for NA on-site, one team added heating unextracted diagnostic samples to obliterate nucleases (HUDSON) to the SHERLOCK system. The system uses heat and chemical reduction to lyse viral particles and destroy interfering ribonucleases found in bodily fluids, allowing a field-deployable virus detection method [23]. However, the system is weighed down due to the need for multiple reagent mixtures. SHINE (SHERLOCK and HUDSON integration to navigate epidemics) combines the two previous systems to detect SARS-CoV-2 in samples
\end{abstract}


Table 3. The most notable CRISPR/Cas-based SARS-CoV-2 detection platforms

\begin{tabular}{|c|c|c|c|c|}
\hline CRISPR system & Cas & Time & Advantages & Disadvantages \\
\hline DETECTR & Cas $12 \mathrm{a}$ & $\begin{array}{l}<40 \\
\min \end{array}$ & $\begin{array}{c}\text { Accurate, easy to use, no thermocyclers, } \\
\text { rapid results }\end{array}$ & $\begin{array}{c}\text { Needs NA extraction; thus, limited } \\
\text { by the quality of extraction kits } \\
\text { and reagents }\end{array}$ \\
\hline AIOD-CRISPR & Cas $12 \mathrm{a}$ & $\begin{array}{l}40 \\
\min \end{array}$ & $\begin{array}{l}\text { Rapid and highly sensitive and specific, } \\
\text { one-pot reaction; thus, no need for } \\
\text { product transferring and less } \\
\text { contamination, results visible with the } \\
\text { naked eye }\end{array}$ & $\begin{array}{l}\text { Needs NA extraction; thus, limited } \\
\text { by the quality and access to } \\
\text { extraction kits and reagents }\end{array}$ \\
\hline $\begin{array}{l}\text { CRISPR/Cas12a- } \\
\text { NER }\end{array}$ & Cas $12 \mathrm{a}$ & $\begin{array}{l}<40 \\
\min \end{array}$ & $\begin{array}{l}\text { Rapid and highly sensitive and specific, } \\
\text { results visible with the naked eye, no } \\
\text { need for sophisticated equipment }\end{array}$ & $\begin{array}{c}\text { Needs NA extraction; thus, limited } \\
\text { by the quality of extraction kits } \\
\text { and reagents }\end{array}$ \\
\hline CRISPR-FDS & Cas $12 \mathrm{a}$ & $\begin{array}{l}<50 \\
\min \end{array}$ & $\begin{array}{l}\text { Rapid and highly sensitive and specific, } \\
\text { no need for sophisticated equipment }\end{array}$ & $\begin{array}{c}\text { Needs NA extraction; thus, limited } \\
\text { by the quality of extraction kits } \\
\text { and reagents }\end{array}$ \\
\hline iSCAN & Cas $12 \mathrm{a}$ & $1 \mathrm{~h}$ & $\begin{array}{l}\text { Rapid and sensitive, easy-to-make } \\
\text { reagents; thus, field-deployable and } \\
\text { proper for large-scale detections }\end{array}$ & $\begin{array}{c}\text { Needs NA extraction; thus, limited } \\
\text { by the quality of extraction kits } \\
\text { and reagents }\end{array}$ \\
\hline VaNGuard & Cas $12 \mathrm{a}$ & $\begin{array}{l}30 \\
\min \end{array}$ & $\begin{array}{c}\text { Rapid and sensitive, robust and } \\
\text { affordable, can tolerate single nucleotide } \\
\text { mutations }\end{array}$ & $x_{0}$ \\
\hline CASdetec & Cas $12 b$ & $1 \mathrm{~h}$ & $\begin{array}{c}\text { Accurate with no cross-reactivity; thus, } \\
\text { less false positive }\end{array}$ & $\begin{array}{c}\text { Needs NA extraction; thus, limited } \\
\text { by the quality of extraction kits } \\
\text { and reagents }\end{array}$ \\
\hline ITP-CRISPR & Cas $12 \mathrm{a}$ & $\begin{array}{c}30 \\
\min \end{array}$ & $\begin{array}{c}\text { Easy to use, automated, uses little } \\
\text { reagents }\end{array}$ & 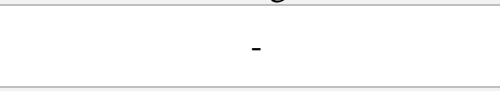 \\
\hline STOPCovid & Cas $12 b$ & $1 \mathrm{~h}$ & $\begin{array}{l}\text { Easy to use, available reagents; thus, } \\
\text { low-cost, no need for NA extraction }\end{array}$ & ${ }^{2}$ \\
\hline CREST & Cas13a & $2 \mathrm{~h}$ & $\begin{array}{l}\text { Sensitive, low-cost, adaptable to large- } \\
\text { scale detection settings, no need for } \\
\text { sophisticated equipment }\end{array}$ & $\begin{array}{c}\text { Needs NA extraction; thus, limited } \\
\text { by the quality of extraction kits } \\
\text { and reagents }\end{array}$ \\
\hline SHINE & Cas13a & $\begin{array}{c}50 \\
\min \end{array}$ & Simple, sensitive, one-step reaction & ( \\
\hline SHERLOCK & Cas $13 a$ & $<1 \mathrm{~h}$ & $\begin{array}{c}\text { Sensitive, rapid, no need for } \\
\text { sophisticated equipment }\end{array}$ & - \\
\hline FELUDA & FnCas9 & $1 \mathrm{~h}$ & $\begin{array}{l}\text { Specific and sensitive, able to detect } \\
\text { single nucleotide mutations }\end{array}$ & 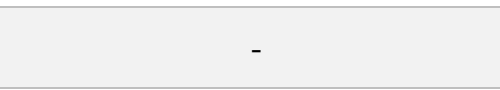 \\
\hline CONAN & Cas3 & $\begin{array}{c}40 \\
\min \end{array}$ & $\begin{array}{l}\text { Rapid, sensitive, low-cost, able to detect } \\
\text { single nucleotide mutations }\end{array}$ & $\begin{array}{c}\text { Needs NA extraction; thus, limited } \\
\text { by the quality of extraction kits } \\
\text { and reagents }\end{array}$ \\
\hline
\end{tabular}

without the need for NA extraction. The device was reported to have a specificity of $100 \%$ and sensitivity of $90 \%$ in 50 patients which is much higher than RT-PCR [33]. The results of the 50 nasopharyngeal samples were visualized by either an in-tube fluorescent or a paper-based colorimetric readout. CREST (Cas13based, rugged, equitable, scalable testing) was developed to further increase the scale and availability of CRISPR-based methods. The system uses accessible reagents and enzymes, cheap thermal cyclers, and easy-to-operate fluorescent detectors. The device is highly sensitive, and while it takes approximately 2 hours to deliver results, it requires no $\mathrm{AC}$ power to operate [34].

It was briefly mentioned that mutations in viruses are not only pivotal in their pathogenesis and escape from the immune system but also in their diagnosis. Viruses are well known to have various mutations due to faulty NA replication. Although most mutations are neutral or deleterious and result in virus death, a few 
of them alter the properties of the virus, such as infectivity, host immunity interaction, and severity of the disease [35]. In the context of virus detection, if the mutations occur in important areas of the virus diagnostic test, they will negatively impact virus detection using RT-PCR or other methods in the sense that CRISPR is unable to bind to the target sequence and false-negative results are reported. It was recently shown that enhanced AsCas12a (enAsCasi2a) as a variant of AsCas12a was able to tolerate single nucleotide variations in the target sequence and was particularly potent in differentiating SARS-CoV-2 from SARS-CoV and MERS-CoV [36]. This VaNGuard system was able to tolerate point mutations in samples.

\section{Miscellaneous CRISPR/Cas-based detection systems}

Although most CRISPR systems utilize Cas12 and Cas13 as their basis of NA recognition and cleavage, there are other CRISPR/Cas systems currently under investigation. In a preprint, a Cas9-based system termed FnCas9 editor linked uniform detection assay (FELUDA) was stated to accurately detect SARS-CoV2 within one hour [37]. Belonging to the class 2 type II, FnCasy is an ortholog of Cas9 derived from Francisella novicida (FnCas9) which can cut DNA. It has been reported that the protein was also highly sensitive in the case of single nucleotide mismatches [38]. It has also been reported that the FELUDA system was not only able to sensitively detect SARS-CoV-2 NA but also differentiate between the target sequences of SARSCoV1 and SARS-CoV-2 that differed in only a single nucleotide [37].

CRISPR/Cas3 is also documented to be used in SARS-CoV-2 detection. Cas3 belongs to class 1 type I category of Cas proteins and it is derived from Escherichia coli and cleaves ssDNA. Yoshimi et al. introduced a Cas3-centered SARS-CoV-2 detection system called Cas3-operated nucleic acid detection $\mathrm{N}$ (CONAN) [39]. CONAN is described to be fast (40 minutes) and low-cost similar to its peers. However, Cas3 capacity to cleave target NA and reporters compared to Cas12 (DETECTR) and Cas13 (SHERLOCK) was unknown until recently. Yoshimi and colleagues compared the specificity of CONAN with DETECTR in 10 positive and 15 negative samples (confirmed via PCR) [39]. The detection rate of both methods was higher than $94 \%$, and the difference was not significant. Table 3 summarizes the most prominent CRISPR/Cas-based modes of SARS-CoV-2 detection.

\section{Conclusion}

The outbreak of COVID-19 has a caused major disruption of our way of life. Specific and sensitive detection of the virus in carriers and asymptomatic patients is pivotal in breaking the transmission cycle of the virus and reducing the infection rates. The advent of gene-editing techniques has brought along the promise of revolutionizing the detection and treatments of our ailments. CRISPR/Cas is the latest and most versatile version of gene-editing platforms, and it can be easily redirected to target a desired genetic sequence. Recently, CRISPR/Cas-based methods have been introduced as rapid, low-cost, sensitive, and specific modes of SARS-CoV-2 detection. Different groups have utilized different Cas proteins, such as Cas3, Cas9, Cas12, and Cas13 to develop a unique system of detection which are on average faster than the conventional RT-PCR with comparable sensitivity. By obviating the need for a thermal cycler machine, CRISPR/Cas provides rapid results by making it desirable in low-income countries without the need for sophisticated equipment. On the other hand, there are some challenges associated with CRISPR/Cas. One downfall of the CRISPR/Cas-based methods is their inability to quantitatively measure the viral load in samples. This is crucial since the level of virus infection is a determinant of disease progression. Furthermore, the limited number of targetable sequences is a hindrance for CRISPR/Cas which allows a restricted number of $g R N A s$ to be engineered. In addition, the target sequence must bear a PAM sequence for the gRNA to complement the target sequence which is not always present. The tolerance for the number and location of mismatches is another aspect of virus detection; the more the mismatches or the closer to the PAM sequence, the less the mismatches are tolerated. CRISPR/Cas13 circumvents this by detecting the target sequence independent of PAM. Also, the inherent capacity of CRISPR/Cas to detect multiple genes by using multiple gRNAs is another benefit of the platform. Overall, CRISPR/Cas systems have many advantages that justify them as POC detection methods of SARS$\mathrm{CoV}-2$ that can be used in the field to lessen the burden 
of the COVID-19 pandemic by providing a rapid and reliable detection platform.

\section{Acknowledgments}

The authors appreciate the efforts of the Tehran University of Medical Sciences in helping with the data gathering.

\section{Author contributions}

SG and HK wrote the first draft of the manuscript. HKand ASN conceived the idea of the manuscript and gathered the data. ASN and HK finalized the draft. All the authors read and approved the finalized manuscript.

\section{Conflict of interests}

None declared.

\section{Ethical declarations}

Not applicable.

\section{Financial support}

No funding was received for this study.

\section{References}

1. Chan JF, Lau SK, To KK, Cheng VC, Woo PC, Yuen KY. Middle East respiratory syndrome coronavirus: another zoonotic betacoronavirus causing SARS-like disease. Clin Microbiol Rev. 2015; 28(2):465-522.

2. Zhou P, Yang XL, Wang XG, Hu B, Zhang L, Zhang W, et al. A pneumonia outbreak associated with a new coronavirus of probable bat origin. Nature. 2020; 579(7798):270-3.

3. Gordon DE, Jang GM, Bouhaddou M, Xu J, Obernier K, White $\mathrm{KM}$, et al. A SARS-CoV-2 protein interaction map reveals targets for drug repurposing. Nature. 2020; 583(7816):459-68.

4. Tešija Kuna A, Hanžek M, Vukasović I, Nikolac Gabaj N, Vidranski V, Ćelap I, et al. Comparison of diagnostic accuracy for eight SARS-CoV-2 serological assays. Biochem Med (Zagreb). 2021; 31(1):010708.

5. Bustin SA, Nolan T. RT-qPCR Testing of SARS-CoV-2: A Primer. Int J Mol Sci. 2020; 21(8):3004.

6. Abbaszadeh-Goudarzi K, Nematollahi MH, Khanbabaei H, Nave HH, Mirzaei HR, Pourghadamyari H, et al. Targeted Delivery of CRISPR/Cas13 as a Promising Therapeutic Approach to Treat SARS-CoV-2. Curr Pharm Biotechnol. 2021; 22(9):1149-55.

7. Shen M, Zhou Y, Ye J, Abdullah Al-Maskri AA, Kang Y, Zeng S, et al. Recent advances and perspectives of nucleic acid detection for coronavirus. J Pharm Anal. 2020; 10(2):97-101.

8. Anantharajah A, Helaers R, Defour JP, Olive N, Kabera F, Croonen L, et al. How to choose the right real-time RT-PCR primer sets for the SARS-CoV-2 genome detection? J Virol Methods. 2021; 295:114197.

9. Jung Y, Park GS, Moon JH, Ku K, Beak SH, Lee CS, et al. Comparative Analysis of Primer-Probe Sets for RT-qPCR of
COVID-19 Causative Virus (SARS-CoV-2). ACS Infect Dis. 2020; 6(9):2513-23.

10. Taylor SC, Laperriere G, Germain H. Droplet Digital PCR versus qPCR for gene expression analysis with low abundant targets: from variable nonsense to publication quality data. Sci Rep. 2017; 7(1):2409.

11. Mautner L, Baillie CK, Herold HM, Volkwein W, Guertler P, Eberle U, et al. Rapid point-of-care detection of SARS-CoV-2 using reverse transcription loop-mediated isothermal amplification (RTLAMP). Virol J. 2020; 17(1):160.

12. Poljak M, Korva M, Knap Gašper N, Fujs Komloš K, Sagadin M, Uršič T, et al. Clinical Evaluation of the cobas SARS-CoV-2 Test and a Diagnostic Platform Switch during 48 Hours in the Midst of the COVID-19 Pandemic. J Clin Microbiol. 2020; 58(6):eo059920.

13. Höhne M, Schreier E. Detection and characterization of norovirus outbreaks in Germany: application of a one-tube RTPCR using a fluorogenic real-time detection system. J Med Virol. 2004; 72(2):312-9.

14. Dong L, Zhou J, Niu C, Wang Q, Pan Y, Sheng S, et al. Highly accurate and sensitive diagnostic detection of SARS-CoV-2 by digital PCR. Talanta. 2021; 224:121726.

15. Suo T, Liu X, Feng J, Guo M, Hu W, Guo D, et al. ddPCR: a more accurate tool for SARS-CoV-2 detection in low viral load specimens. Emerg Microbes Infect. 2020; 9(1):1259-68.

16. Qasem A, Shaw AM, Elkamel E, Naser SA. Coronavirus Disease 2019 (COVID-19) Diagnostic Tools: A Focus on Detection Technologies and Limitations. Curr Issues Mol Biol. 2021; 43(2):728-48.

17. Hou T, Zeng W, Yang M, Chen W, Ren L, Ai J, et al. Development and evaluation of a rapid CRISPR-based diagnostic for COVID-19. PLoS Pathog. 2020; 16(8):e1008705.

18. Tsou JH, Liu H, Stass SA, Jiang F. Rapid and Sensitive Detection of SARS-CoV-2 Using Clustered Regularly Interspaced Short Palindromic Repeats. Biomedicines. 2021; 9(3):239.

19. Ishino $Y$, Shinagawa H, Makino K, Amemura M, Nakata A. Nucleotide sequence of the iap gene, responsible for alkaline phosphatase isozyme conversion in Escherichia coli, and identification of the gene product. J Bacteriol. 1987; 169(12):542933.

20. Xu Y, Li Z. CRISPR-Cas systems: Overview, innovations and applications in human disease research and gene therapy. Comput Struct Biotechnol J. 2020; 18:2401-15.

21. Hu Y, Zhou Y, Zhang M, Ge W, Li Y, Yang L, et al. CRISPR/Cas9-Engineered Universal CD19/CD22 Dual-Targeted CAR-T Cell Therapy for Relapsed/Refractory B-cell Acute Lymphoblastic Leukemia. Clin Cancer Res. 2021; 27(10):2764-72. 22. Jayamohan H, Lambert CJ, Sant HJ, Jafek A, Patel D, Feng H, et al. SARS-CoV-2 pandemic: a review of molecular diagnostic tools including sample collection and commercial response with associated advantages and limitations. Anal Bioanal Chem. 2021; 413(1):49-71.

23. Myhrvold C, Freije CA, Gootenberg JS, Abudayyeh OO, Metsky $\mathrm{HC}$, Durbin AF, et al. Field-deployable viral diagnostics using CRISPR-Cas13. Science. 2018; 360(6387):444-8.

24. Broughton JP, Deng X, Yu G, Fasching CL, Servellita V, Singh $\mathrm{J}$, et al. CRISPR-Cas12-based detection of SARS-CoV-2. Nat Biotechnol. 2020; 38(7):870-4. 


\section{Ghaffari et al.}

25. Ding X, Yin K, Li Z, Lalla RV, Ballesteros E, Sfeir MM, et al. Ultrasensitive and visual detection of SARS-CoV-2 using all-in-one dual CRISPR-Cas12a assay. Nat Commun. 2020; 11(1):4711.

26. Wang X, Zhong M, Liu Y, Ma P, Dang L, Meng Q, et al. Rapid and sensitive detection of COVID-19 using CRISPR/Cas12a-based detection with naked eye readout, CRISPR/Cas12a-NER. Sci Bull (Beijing). 2020; 65(17):1436-9.

27. Huang Z, Tian D, Liu Y, Lin Z, Lyon CJ, Lai W, et al. Ultrasensitive and high-throughput CRISPR-p owered COVID-19 diagnosis. Biosens Bioelectron. 2020; 164:112316.

28. Ali Z, Aman R, Mahas A, Rao GS, Tehseen M, Marsic T, et al. iSCAN: An RT-LAMP-coupled CRISPR-Cas12 module for rapid, sensitive detection of SARS-CoV-2. Virus Res. 2020; 288:198129.

29. Teng F, Guo L, Cui T, Wang XG, Xu K, Gao Q, et al. CDetection: CRISPR-Cas12b-based DNA detection with sub-attomolar sensitivity and single-base specificity. Genome Biol. 2019; 20(1):132.

30. Rahimi H, Salehiabar M, Barsbay M, Ghaffarlou M, Kavetskyy T, Sharafi A, et al. CRISPR Systems for COVID-19 Diagnosis. ACS Sens. 2021; 6(4):1430-45.

31. Brandsma E, Verhagen H, van de Laar TJW, Claas ECJ, Cornelissen M, van den Akker E. Rapid, Sensitive, and Specific Severe Acute Respiratory Syndrome Coronavirus 2 Detection: A Multicenter Comparison Between Standard Quantitative ReverseTranscriptase Polymerase Chain Reaction and CRISPR-Based DETECTR. J Infect Dis. 2021; 223(2):206-13.

32. Ramachandran A, Huyke DA, Sharma E, Sahoo MK, Huang C, Banaei N, et al. Electric field-driven microfluidics for rapid CRISPR-based diagnostics and its application to detection of SARS-CoV-2. Proc Natl Acad Sci U S A. 2020; 117(47):29518-25.

33. Arizti-Sanz J, Freije CA, Stanton AC, Boehm CK, Petros BA, Siddiqui S, et al. Integrated sample inactivation, amplification, and Cas13-based detection of SARS-CoV-2. bioRxiv. 2020. [Preprint]. 34. Rauch JN, Valois E, Solley SC, Braig F, Lach RS, Audouard M, et al. A Scalable, Easy-to-Deploy Protocol for Cas13-Based Detection of SARS-CoV-2 Genetic Material. J Clin Microbiol. 2021; 59(4):e02402-20.

35. Harvey WT, Carabelli AM, Jackson B, Gupta RK, Thomson EC, Harrison EM, et al. SARS-CoV-2 variants, spike mutations and immune escape. Nat Rev Microbiol. 2021; 19(7):409-24.

36. Ooi KH, Liu MM, Tay JWD, Teo SY, Kaewsapsak P, Jin S, et al. An engineered CRISPR-Cas12a variant and DNA-RNA hybrid guides enable robust and rapid COVID-19 testing. Nat Commun. 2021; 12(1):1739.

37. Azhar M, Phutela R, Ansari AH, Sinha D, Sharma N, Kumar M, et al. Rapid, field-deployable nucleobase detection and identification using FnCas9. bioRxiv. 2020:2020.04.07.028167.

38. Acharya S, Mishra A, Paul D, Ansari AH, Azhar M, Kumar M, et al. Francisella novicida Cas9 interrogates genomic DNA with very high specificity and can be used for mammalian genome editing. Proc Natl Acad Sci U S A. 2019; 116(42):20959-68.

39. Yoshimi K, Takeshita K, Yamayoshi S, Shibumura S, Yamauchi Y, Yamamoto M, et al. Rapid and accurate detection of novel coronavirus SARS-CoV-2 using CRISPR-Cas3. medRxiv. 2020. [Preprint]. 